\title{
Escalas de Estilos Interpessoais (ESEI): Construção, Validade Fatorial e Consistência Interna
}

\author{
Interpersonal Styles Scales (ISS): Construction, Factorial Validity \\ and Internal Consistency
}

\author{
Mauro de Oliveira Magalhães* \\ Universidade Federal da Bahia, Salvador, Bahia, Brasil
}

\begin{abstract}
Resumo
Este artigo descreve a construção e validação inicial do Inventário de Estilos Interpessoais (ESEI). $\mathrm{O}$ estilo interpessoal é um construto multidimensional definido como a maneira que o indivíduo busca por aceitação e status social. Uma amostra de 984 sujeitos com idades entre 18 e 63 anos, 395 homens e 589 mulheres, responderam ao ESEI. A análise fatorial exploratória revelou uma estrutura de oito fatores consistente com as categorias teóricas de estilo interpessoal descritas na literatura. Os oito fatores explicaram $46,24 \%$ da variância total e os índices de consistência interna das subescalas variaram entre bons e excelentes. Os resultados sugerem que o ESEI é um instrumento promissor para uso em pesquisa e contextos aplicados, embora sejam necessárias mais evidências de validade. Palavras-chave: Estilos interpessoais, personalidade, avaliação psicológica.
\end{abstract}

\begin{abstract}
This paper describes the construction and initial validation of the Interpersonal Style Inventory (ISI). Interpersonal style is a multidimensional construct defined as the way an individual look for acceptance and social status. A sample of 984 subjects with ages ranging from 18 to 63 years, 395 men and 589 women, answered the ISI. Exploratory factorial analysis revealed an eight factor structure consistent with theoretical categories of interpersonal style described in the literature. The eight factors explained $46.2 \%$ of total variance, and internal consistency indexes for the subscales ranged from good to excellent. Results suggest that ISI is a promising tool for using in research and applied settings, though, further evidence of its validity is still necessary.

Keywords: Interpersonal styles, personality, psychological assessment.
\end{abstract}

O enfoque interpessoal da personalidade tem início com as ideias de Adler (1907/2005). O autor definiu a personalidade, ou 'estilo pessoal', como um movimento integrado e singular de busca por aceitação e status social, uma concepção compartilhada por autores contemporâneos (Roberts \& Wood, 2006). A seguir, Sullivan (1953) apresentou a sua teoria interpessoal do comportamento. Para Sullivan (1953), o ser humano está primariamente motivado para obter segurança, manter sua autoestima e evitar sentimentos de ansiedade que podem resultar das dificuldades nas relações interpessoais. Ele afirmou que a ansiedade tem origem na insegurança que pode advir da dependência interpessoal prolongada da criança em relação a seus cuidadores. As formas características de o indivíduo dominar a sua ansiedade foram denominadas operações de segurança (Sullivan, 1953) ou reflexos interpessoais (Leary, 1957/2004). A obra pioneira de

* Endereço para correspondência: Universidade Federal da Bahia, Instituto de Psicologia, São Lázaro, Federação, Salvador, BA, Brasil 40170-115. E-mail: mauro.m@ terra.com.br
Leary (1957/2004) defendeu ostensivamente que, para compreender um indivíduo, é primordial obter informação sobre seus relacionamentos sociais, mais especificamente sobre suas técnicas e estratégias recorrentes para evitar e/ou lidar com a ansiedade e as reações que evoca nas demais pessoas. Nesta visão, a personalidade é um conjunto organizado destes reflexos interpessoais que se tornam recorrentes nas interações sociais de um indivíduo e caracterizam o seu estilo interpessoal.

Kiesler (1996; Kiesler \& Schmidt, 2006) segue este argumento e defende que o comportamento humano somente pode ser compreendido em relação aos seus contextos interpessoais pregressos e atuais. Assim como Leary (1957/2004), Kiesler (1996) afirma que todo comportamento interpessoal busca evitar a ansiedade e realçar a autoestima. $\mathrm{O}$ autor considera que a avaliação do estilo interpessoal requer uma ampla coleção de medidas específicas que sejam sistematicamente relacionadas entre si a fim de identificar o padrão de comportamentos sociais que o indivíduo utiliza.

Embora existam algumas diferenças entre teorias interpessoais da personalidade, suas premissas convergem 
ao propor que as dimensões básicas do comportamento interpessoal são o controle (dominância versus submissão) e a necessidade de afiliação (hostilidade versus amizade) (Kiesler \& Schmidt, 2006; Leary, 1957/2004). O comportamento interpessoal reflete combinações específicas de inclinações pertinentes a estas duas dimensões. Neste sentido, Digman (1997) concluiu que dois fatores de ordem mais elevada organizam o modelo dos cinco grandes fatores. $\mathrm{O}$ autor percebeu que estes dois fatores correspondem a dicotomias já conhecidas, tais como interesse social e busca de superioridade (Adler, 1907/2005), união e individualismo (Rank, 1945), amor e poder (Leary, 1957/2004), agência e comunhão (Bakan, 1966; Horowitz, 2004; Wiggins, 1991), status e popularidade (Hogan, 1982); e intimidade e poder (McAdams, 1985).

As polaridades afiliação/comunhão e poder/agência (ou conceitos correlatos) têm guiado esforços para avaliar o comportamento interpessoal (Gurtman, 2009; Leary, 1957/2004; Pincus \& Gurtman, 2006; Wiggins, 1991). Leary (1957/2004) concluiu que seria útil organizar estas duas dimensões básicas em um círculo, que denominou círculo interpessoal. Deste modo, desenvolveu o Interpersonal CheckList - ICL, composto por oito escalas interpessoais situadas em dois eixos: o vertical formado pelos pólos dominância e submissão, e o horizontal por amabilidade e hostilidade. Este tipo de modelo, atualmente denominado circumplexo, assume que cada inclinação interpessoal é uma mistura particular destas inclinações dicotômicas. O posicionamento de um indivíduo próximo a um dos pólos destes eixos significa que ele irá apresentar comportamentos ou intenções específicas de amabilidade ou hostilidade, de dominância ou submissão.

Inspirados na obra de Leary (1957/2004), outros modelos circumplexos foram desenvolvidos. O IAS-R $-I n-$ terpersonal Adjective Scales Revised (Wiggins, 1995) e o IMI-C - Octant Scale Impact Message Inventory (Kiesler \& Schmidt, 2006) estão entre os testes de modelo circumplexo mais utilizados (Gurtman, 2009). Dada a similaridade conceitual entre os diversos modelos circumplexos, Gurtman (2009) propôs rótulos genéricos para as categorias teóricas de estilo interpessoal utilizadas nos estudos da área que, na verdade, foram os mesmos utilizados por Kiesler e Schmidt (2006). Estes rótulos definem oito categorias de comportamento em quatro dimensões bipolares, a saber: dominante e submisso, hostil-dominante e amigável-submisso, hostil e amigável, hostil-submisso e amigável-dominante.

Os vetores afiliação e poder, cujas combinações constituem as categorias de estilo interpessoal de modelos circumplexos, estão presentes na teoria clássica da motivação de David McClelland (1985), que permanece influente em diversos domínios da psicologia (Boyatzis, 2008; Rego $\&$ Leite, 2003). O motivo de poder refere-se ao desejo de influenciar e controlar os demais, associado a atividades de competição, controle de recursos e aquisição de status, correspondendo à polaridade dominância versus submissão. O motivo afiliativo refere-se à busca de relacionamentos amigáveis e cooperativos, correspondendo à polaridade hostilidade versus amizade (Rego \& Leite, 2003). Porém, modelos circumplexos não apresentam de modo claro a motivação de realização, uma terceira categoria motivacional descrita por McClelland (1985) que se destaca na pesquisa em psicologia da personalidade (Bipp, Steinmayr, \& Spinath, 2008; Pang, Villacorte, Chin, \& Morrison, 2009). O motivo de realização refere-se ao interesse por apresentar um desempenho diferenciado e/ou valorizado, onde o indivíduo contribui para o progresso e/ou bem-estar de sua comunidade através das suas realizações pessoais, atendendo ou ultrapassando padrões e expectativas sociais (McClelland \& Koestner, 1992). Este tipo de motivação pode ser encontrado nas formulações de Adler (1907/2005) sobre os estilos interpessoais.

Adler (1907/2005) descreveu quatro inclinações genéricas de estilos: (a) o estilo socialmente interessado, que procura estar a serviço da comunidade; (b) o dominador, que deseja impor sua vontade; (c) o obtentor, que busca ter suas necessidades atendidas pelos demais; e (d) o evitador, que evade das demandas da vida por receio de ser derrotado e humilhado por outros. A descrição de Adler (1907/2005) de um estilo dominador já possui representatividade nos modelos circumplexos, evidenciada no eixo bipolar dominância e submissão. No entanto, observa-se que o estilo socialmente interessado envolve não somente uma disposição favorável para afiliação ou proximidade interpessoal, destacada em modelos circumplexos, mas um propósito de contribuição ou realização alinhada com demandas coletivas. Este propósito ou motivação mostra-se enfraquecido nos estilos evitador e obtentor. O primeiro esquiva-se de responsabilidades por receio do fracasso, e o segundo deseja ser cuidado pelas outras pessoas. Ambos carecem da motivação para contribuir de forma ativa para a sua coletividade através de suas realizações pessoais. Em contraste, o estilo socialmente interessado se revela no esforço do indivíduo para oferecer contribuições à vida comum. Portanto, as formulações de Adler (1907/2005) incluem não somente as motivações de afiliação e poder descritas em modelos circumplexos, mas também o motivo de realização, posteriormente mais bem definido e explorado na obra de McClelland (1985) e de pesquisadores contemporâneos (por exemplo, Pang et al., 2009).

Gurtman (2009) salientou que é perfeitamente possível avaliar e analisar estilos interpessoais sem encaixá-los em modelos circumplexos. O imprescindível é que as medidas avaliem as diversas qualidades de esforços individuais para obter aceitação e status social. O Inventário BASIS-A (Basic Adlerian Scales of Interpersonal Style; Curlette, Kern, \& Wheeler, 1997) não obedece a uma estrutura circumplexa e tem revelado ampla utilidade em pesquisa e intervenção (Kern, Gormley, \& Curlette, 2008). A base conceitual das escalas do BASIS-A foi a psicologia individual de Alfred Adler. Para Adler (1907/2005), o estilo interpessoal se forma a partir das percepções da criança sobre suas interações sociais na família, na escola e entre seus pares. O autor defende que a criança, ao interpretar estas primeiras experiências, desenvolve um sistema de 
Magalhães, M. O. (2013). Escalas de Estilos Interpessoais (ESEI): Construção, Validade Fatorial e Consistência Interna.

crenças sobre as relações sociais e uma maneira consistente de agir que permanece na vida adulta. Este será o modus operandi individual, ou estilo interpessoal, para enfrentamento das demandas da convivência com objetivos de obter aceitação e status social.

Neste sentido, os itens do BASIS-A são sentenças descritoras de comportamentos interpessoais na infância. O cabeçalho do instrumento dita o início de todas as sentenças com a introdução "Quando eu era criança, eu...", que tem continuidade na descrição de comportamentos específicos. Assim como no BASIS-A, a construção dos itens que compõem as escalas de estilos interpessoais do Inventário ESEI, objeto deste estudo, utilizou a percepção do indivíduo sobre a frequência de determinados comportamentos interpessoais na infância. Portanto, a criação destes instrumentos foi baseada no uso da memória autobiográfica na avaliação da personalidade (Adler, 1907/2005; Bruhn, 1995), cujos conceitos e evidências de validade serão apresentados a seguir.

\section{Memória Autobiográfica e Avaliação da Personalidade}

As pesquisas no campo da memória humana compõem um mosaico de perspectivas teóricas, às vezes contrastantes e conflituosas. No entanto, pesquisadores concordam em algumas premissas básicas. Entre as menos controversas, e talvez a mais importante, é que a memória não é o registro fiel de acontecimentos; ao contrário, a memória é um processo permeado de erros, ilusões, distorções e, por que não dizer, criatividade (Tulving, 2002). Nesta perspectiva, a memória consiste em esquemas sobre o mundo, mais do que traços ou imagens; e registros mnemônicos são atitudes e crenças derivadas das experiências passadas, mais do que o acúmulo de informações. Estas atitudes, crenças e questões estão ativas no presente; e um processo de justificação regula a reconstrução dos eventos passados para que os 'fatos' se apresentem consistentes com a atitude original do processo de recordação. Estas ideias são compartilhadas por inúmeros autores (Bruhn, 1995; McAdams, 1985; Neisser, 1982; Pillemer, 2003; Schacter \& Addis, 2007; Tulving, 2005) e estão presentes na obra pioneira de Adler (1931/2010). O autor destacou a função criadora de sentido dos processos de memória, onde o indivíduo seleciona e organiza impressões do passado de forma a articular uma narrativa pessoal que ofereça identidade, razão e propósito para ações no presente.

Durante a última década estudos com uso de recursos de neuro-imagem resultaram em avanços importantes na neurociência da memória construtiva (ver revisão de Schacter \& Slotnick, 2004). A atualidade das ideias de Adler destaca-se na constatação que a memória não está somente relacionada à capacidade de re-experienciar eventos passados, mas também a capacidade de imaginar, simular ou pré-experienciar episódios no futuro (Atance \& O’Neill, 2005; Buckner \& Carroll, 2007; Schacter \& Addis, 2007; Suddendorf \& Busby, 2005; Tulving, 2005).
A adequada simulação de episódios futuros, com vistas a preparar o indivíduo para enfrentá-los em sua novidade, requer um sistema capaz de extrair e recombinar elementos de experiências passadas num processo mais construtivo do que reprodutivo. De acordo com Tulving (2002, 2005), fazer estas conexões significa projetar-se tanto no passado quanto no futuro, tal como uma 'viagem mental no tempo'.

Autores envolvidos na aplicação clínica da memória autobiográfica concordam que as recordações de infância se referem a "questões básicas não resolvidas ou tarefas inacabadas prioritárias na agenda pessoal do indivíduo" (Bruhn, 1995, p. 596); sendo "a nossa reconstrução das experiências da primeira infância não somente consistente com as nossas crenças presentes, mas a maneira pela qual resgatamos estes eventos reforça o que acreditamos hoje" (De Muth \& Bruhn, 1997, p. 25). Portanto, estas lembranças podem ser entendidas como construções idiossincráticas do indivíduo, reveladoras das suas crenças e atitudes particulares ainda ativas no presente.

Perguntas sobre lembranças infantis possuem vantagens importantes em relação a questões sobre comportamentos ou atitudes atuais do respondente. Ao se referirem a eventos da infância, reduzem a defensividade que pode emergir diante da possibilidade de relatar comportamentos considerados negativos ou inadequados na vida presente. As pessoas estão mais dispostas a admitir características negativas se estas são localizadas num 'passado distante' da infância (Curlette et al., 1997).

Este trabalho irá apresentar a construção de um instrumento de avaliação de estilos interpessoais, o Inventário ESEI (Escalas de Estilos Interpessoais). Esta avaliação se dará por meio das percepções do indivíduo sobre seus comportamentos sociais na infância. Acredita-se que esta percepção reflete crenças e atitudes ativas nas manifestações comportamentais presentes, conferindo-lhes a consistência característica de um estilo interpessoal. Portanto, a construção do Inventário ESEI insere-se numa tradição de pesquisa sobre o uso da memória autobiográfica na avaliação da personalidade, tendo como modelo o Inventário BASIS-A (Curlette et al., 1997).

As categorias de estilo interpessoal descritas na literatura (Kiesler \& Schmidt, 2006; Leary, 1957/2004; Wiggins, 1991, 1995) e as ideias seminais de Adler (1907/2005) foram utilizadas como guias conceituais para a elaboração dos itens das escalas do Inventário ESEI. Num primeiro momento, os rótulos genéricos propostos por Gurtman (2009) foram utilizados para nomear as categorias de estilo interpessoal construídas a partir da revisão teórica de modelos circumplexos. Estes rótulos foram considerados úteis como sínteses das definições conceituais a serem operacionalizadas. Além disto, os estilos socialmente interessado, obtentor e evitador, tal como descritos por Adler (1907/2005), também foram utilizados como guia conceitual para a formulação de itens, pois se considera que não estejam claramente representados nos modelos circumplexos. Na descrição destes estilos, Adler (1907/2005) incluiu variações em motivações de realização, um aspecto 
relevante para a conquista de aceitação e status social na cultura contemporânea. Por outro lado, o estilo dominador, também descrito pelo autor, já possui representatividade nos modelos circumplexos, evidenciada no eixo bipolar dominância e submissão. A construção do Inventário ESEI não teve, a priori, o objetivo de reproduzir um modelo circumplexo, mas de oferecer um conjunto abrangente e teoricamente integrado de medidas de estilo interpessoal.

\section{Método}

\section{Participantes}

Em uma primeira etapa, este estudo contou com a participação de quatro juízes conhecedores da literatura relacionada à base conceitual do instrumento. A seguir, uma amostra de 30 estudantes universitários com idades entre 18 e 25 anos responderam ao instrumento a fim de testar a compreensão dos itens. Por fim, uma amostra de 984 sujeitos com idades entre 18 e 63 anos $(M=29 ; D P=$ 4,5), 395 homens e 589 mulheres, residentes nas regiões sul, sudeste e nordeste do Brasil, com escolaridade mínima de ensino médio completo, respondeu ao instrumento em sua versão final.

\section{Instrumento}

A formulação de 118 itens descritores de comportamentos sociais na infância foi feita de modo a representar os temas interpessoais recorrentes na literatura. Foram construídos itens representativos das oito categorias de comportamento comuns aos vários modelos circumplexos do domínio interpessoal da personalidade, nomeadas por Gurtman (2009), a saber: dominante (ex.: "gostava de tomar decisões pelo grupo"); hostil-dominante (ex.: "não baixava a cabeça para ninguém"); hostil (ex.: "vingava-me quando me sentia prejudicado"); hostil-submisso (ex.: "chorava quando contrariado"); submisso (ex.: "comportava-me bem para ser aceito"); amigável-submisso (ex.: "era disciplinado"); amigável (ex.: "não me importava de emprestar meus brinquedos a outras crianças") e amigável-dominante (ex.: "queria ser o mais popular da turma"). E também foram operacionalizadas as categorias socialmente interessado (ex.: "gostava de colaborar nas tarefas da casa"), obtentor (ex.: "os adultos ou mais velhos faziam todas as minhas vontades") e evitador (ex.: "preferia passar despercebido") descritas por Adler (1907/2005).

As instruções para o respondente foram as seguintes:

As afirmativas abaixo são sobre seus comportamentos quando criança nas relações familiares, na escola e no grupo de amigos. Quando você responder, compare-se com seus irmãos, irmãs ou amigos, antes de você completar dez anos. A palavra "pais" inclui a pessoa ou pessoas que cuidaram de você naquela época. Responda cada item o mais honestamente que você puder.

$\mathrm{O}$ início de todas as sentenças possui a introdução "Quando eu era criança, eu ....", que tem continuidade na descrição de comportamentos específicos. O respondente é convidado a marcar numa escala Likert de cinco pontos, desde discordo totalmente (1) até concordo totalmente (5), o seu grau de concordância com a descrição comportamental oferecida em cada frase.

A análise dos juízes foi realizada através de um formulário contendo as definições conceituais das categorias de estilo interpessoal, os itens propostos e as instruções para avaliação. A avaliação de cada item incluiu os seguintes aspectos: (a) adequação do conteúdo, (b) a clareza da formulação, (c) a relevância, e (d) identificação da categoria de estilo interpessoal. $O$ formulário também incluiu espaço para que os juízes apresentassem sugestões.

\section{Procedimentos}

Os formulários de avaliação preenchidos pelos juízes foram analisados considerando cada critério em separado, bem como as sugestões de reformulação apresentadas. A manutenção do item na escala foi condicionada a obtenção de concordância entre, pelo menos, três juízes em cada um dos quesitos.

O teste de compreensão semântica foi realizado mediante aplicação do instrumento em universitários de diversos cursos de graduação matriculados em uma disciplina de metodologia científica. Após esclarecimento, os participantes responderam ao instrumento e julgaram as instruções e itens em termos de sua inteligibilidade, que foi considerada satisfatória.

A terceira etapa de coleta de dados foi realizada em instituições de ensino e organizações de trabalho, após autorização das instâncias responsáveis e consentimento dos participantes. As aplicações foram predominantemente coletivas. Estes procedimentos foram realizados mediante aprovação do Comitê de Ética da instituição de afiliação do pesquisador por ocasião da pesquisa. Foram atendidas as determinações éticas da resolução 196/1996 do Conselho Nacional de Saúde sobre a pesquisa com seres humanos.

Os dados foram examinados em termos de adequação dos dados a procedimentos de análise fatorial. A adequação da amostra foi verificada pelo determinante da matriz de correlação, pelo coeficiente de adequação da amostra de Kaiser-Meyer-Olkin (KMO), e pelo teste de esfericidade de Bartlett. Assume-se que as escalas propostas são correlacionadas, considerando a característica circumplexa das relações teóricas entre os fatores. Portanto, foi utilizada a rotação Direct Oblimin, técnica mais adequada para investigar possíveis sobreposições entre fatores e evitar a perda de informação relevante (Costello \& Osborne, 2005). A precisão de cada escala de estilo interpessoal foi avaliada por alfas de Cronbach. Foi examinada a contribuição de cada item para a precisão de sua respectiva escala.

\section{Resultados e Discussão}

A avaliação feita pelos juízes resultou na exclusão de 14 itens considerados pouco adequados em mais de um critério adotado. Entre os itens restantes, 23 não alcançaram concordância de $80 \%$ em termos de categorização em determinada dimensão de estilo interpessoal. No entanto, 
Magalhães, M. O. (2013). Escalas de Estilos Interpessoais (ESEI): Construção, Validade Fatorial e Consistência Interna.

entre estes, 10 foram considerados itens pertinentes, claros e bastante relevantes para os objetivos do instrumento. Sendo assim, optou-se pela manutenção dos mesmos. Ao final, permaneceram 91 itens.

$\mathrm{Na}$ análise fatorial exploratória preliminar, o gráfico Scree sugeriu 9 fatores como a melhor explicação possível para os dados apresentados. A análise de componentes principais foi seguida por rotação Direct Oblimin e revelou a inadequação de 8 itens, que não contribuíram para uma estrutura fatorial bem delimitada, pois não atenderam os critérios mínimos de obter uma carga fatorial primária acima de $0,40 \mathrm{em}$ determinado fator e não apresentar cargas fatoriais acima de 0,40 em mais de um fator. A análise posterior com a retirada destes itens é descrita a seguir.

$\mathrm{O}$ coeficiente Kaiser-Meyer-Olkin $(\mathrm{KMO}=0,908)$, o determinante da matriz de correlações (4,29E-016), o teste de esfericidade de Bartlett $\left[\mathrm{c}^{2}(3403)=620644,121 ; p<\right.$ $0,01]$ revelaram adequação satisfatória dos dados à análise fatorial. A diagonal da matriz de correlação de anti-imagem recomendou a retenção de todos os itens para análise (MSA $>0,80$ ); e as comunalidades foram todas acima de 0,40 . Portanto, considerando estes indicadores, a análise fatorial foi conduzida com todos os 83 itens selecionados.

O gráfico Scree sugeriu 8 ou 9 fatores como explicações possíveis para os dados apresentados. Em primeiro lugar foi examinada a solução com oito fatores, que obtiveram autovalores adequados (eigenvalue $\geq 1,70$ ) e explicaram $46,24 \%$ da variância. A análise de componentes principais seguida por rotação Direct Oblimin resultou numa distribuição satisfatória dos itens entre os oito fatores, tanto em termos de cargas fatoriais quanto de aderência à teoria.
A Tabela 1 apresenta os resultados da solução de oito fatores, com os respectivos autovalores e variância explicada por cada fator. Também estão incluídos os índices de precisão (alfas de Cronbach) das escalas formadas pelos itens de cada fator. O exame da alteração dos alfas se cada item deletado revelou que todos os itens contribuem para a precisão de sua respectiva escala. A caracterização teórica dos itens componentes de cada fator foi sintetizada em rótulos correspondentes e incluída na Tabela 1, explicados mais adiante.

$\mathrm{Na}$ solução com nove fatores, os autovalores foram adequados (eigenvalue $\geq 1,70$ ) e explicaram $48,29 \%$ da variância. $\mathrm{O}$ nono fator foi formado por 6 itens que também obtiveram cargas superiores a 0,4 no terceiro fator. A identidade teórica dos 6 itens sugere que compõem uma faceta do terceiro fator. Sendo assim, foi realizada uma análise fatorial com os 15 itens do terceiro fator, a fim de explorar a existência de facetas componentes. Esta análise resultou em uma solução de dois fatores, que explicaram $47,10 \%$ da variância com autovalores 5,36 (35,74\%) e 1,71 $(11,37 \%)$. No primeiro agruparam-se 8 itens com cargas fatoriais entre 0,48 e 0,72 . E no segundo 7 itens com cargas entre 0,47 e 0,85 . Somente um item apresentou cargas acima de 0,3 nos dois fatores. Estas cargas fatoriais foram 0,475 e 0,301 . Portanto, a magnitude da diferença entre as cargas recomendou a sua permanência no fator com carga mais elevada. A análise de consistência interna resultou em alfas de Cronbach 0,82 e 0,81 para o primeiro e segundo fator, respectivamente. Portanto, os dados sugerem que duas facetas compõem o terceiro fator, cujos significados teóricos serão explorados adiante.

Tabela 1

Exemplos de Item, Variância Explicada, Autovalores e Alfas de Cronbach das Escalas de Estilo Interpessoal

\begin{tabular}{|c|c|c|c|}
\hline Dimensão de Estilo Interpessoal (Numero de itens) & \% variância explicada & Autovalores & Alfa de Cronbach \\
\hline \multicolumn{4}{|l|}{ "Quando eu era criança eu ....". } \\
\hline $\begin{array}{l}\text { Fator 1: Cautela (13) } \\
\text { “... achava que não gostavam de mim”. }\end{array}$ & 12,70 & 10,54 & 0,85 \\
\hline $\begin{array}{l}\text { Fator 2: Comando (12) } \\
\text { “... gostava de tomar decisões pelo grupo". }\end{array}$ & 10,91 & 9,05 & 0,91 \\
\hline $\begin{array}{l}\text { Fator 3: Reconhecimento (15) } \\
\text { "... gostava de mostrar minha inteligência". }\end{array}$ & 7,88 & 6,54 & 0,90 \\
\hline $\begin{array}{l}\text { Fator 4: Intitulação (10) } \\
\text { "... atendiam a tudo o que eu pedia". }\end{array}$ & 4,23 & 3,51 & 0,84 \\
\hline $\begin{array}{l}\text { Fator 5: Superioridade (5) } \\
\text { “... preferia a companhia dos adultos". }\end{array}$ & 3,17 & 2,63 & 0,71 \\
\hline $\begin{array}{l}\text { Fator 6: Confrontação ( } 8 \text { ) } \\
\text { "... vingava-me quando me sentia prejudicado". }\end{array}$ & 2,81 & 2,34 & 0,80 \\
\hline $\begin{array}{l}\text { Fator 7: Conformidade (6) } \\
\text { "... era obediente". }\end{array}$ & 2,39 & 1,98 & 0,70 \\
\hline $\begin{array}{l}\text { Fator 8: Pertencimento (13) } \\
\text { ". . . fazia amigos com facilidade". }\end{array}$ & 2,16 & 1,80 & 0,86 \\
\hline Total & $46,24 \%$ & 38,40 & ----- \\
\hline
\end{tabular}


A análise do conteúdo dos itens de cada fator possibilitou identificar dimensões de estilo interpessoal com identidades teóricas relevantes e aderência satisfatória a base conceitual adotada. $\mathrm{O}$ primeiro fator agrupou doze itens associados às categorias de estilo hostil-submisso, hostil, e evitador. Observou-se consistência de conteúdo entre as frases, que descrevem sentimentos de inadequação, vitimização e ressentimento em relação a outras pessoas, principalmente figuras de autoridade. No Inventário ESEI, este fator será denominado Cautela (CA). Acredita-se que escores elevados nesta escala signifiquem uma percepção desfavorável do ambiente social que, por sua vez, resulta em comportamentos de vigilância interpessoal, desconfiança, e ansiedade que pode desencadear comportamentos agressivos ou de isolamento social.

O segundo fator revelou acentuada consistência interna entre doze itens descritores do desejo de dominar e/ou comandar os acontecimentos sociais, relacionados às categorias dominante e amigável-dominante. No Inventário ESEI, este fator será denominado Comando (CM), onde se espera que quanto mais elevados os escores tanto mais o estilo interpessoal se caracterizará pelo desejo de liderar e/ou controlar as decisões e ações do grupo.

$\mathrm{O}$ terceiro fator agrupou quinze itens que descrevem comportamentos de busca do reconhecimento externo de figuras de autoridade e/ou aquisição de status através do comportamento adequado ou desempenho diferenciado na realização de tarefas relacionadas às expectativas sociais. Os itens agrupados incluem formulações pertencentes às categorias amigável-dominante, amigável-submisso e socialmente interessado. O conjunto de itens caracteriza comportamentos de aquisição de status ou aprovação social através do atendimento de expectativas externas. No Inventário ESEI, este fator será denominado Busca de reconhecimento (BR), onde se espera que quanto mais elevados os escores tanto mais o indivíduo estará preocupado em apresentar comportamentos que resultem na aprovação externa e/ou na conquista de atenção social através do atendimento ou superação de expectativas de desempenho.

Este fator demonstrou ser composto de duas facetas. A primeira faceta mostrou consistência de conteúdo em torno do tema de aquisição de status através do desempenho ou apresentação de talentos especiais, e foi composta por oito itens pertinentes as categorias amigável-dominante e socialmente interessado. Sendo assim, esta faceta será denominada de Busca de Sucesso (BSU). A segunda faceta foi composta por sete itens formulados para as categorias submisso e amigável-submisso, descritores do desejo de obter aprovação de outras pessoas através de comportamentos adequados as expectativas externas. Esta faceta será denominada de Busca de Aprovação (BAP).

$\mathrm{O}$ quarto fator agrupou dez itens que descrevem comportamentos de uso ou simulação de vulnerabilidade pessoal para despertar sentimentos de compaixão em outras pessoas a fim de obter privilégios ou o apoio externo para o atendimento das próprias necessidades. Entre os dez itens que carregaram neste fator, a frase “. . . usava minha fragilidade para obter o que desejava dos outros" ilustra esta categoria de estilo interpessoal. Este fator não encontra uma correspondência clara nos modelos circumplexos, mas foi descrita por Adler (1907/2005), que a denominou de estilo obtentor, caracterizado como o uso pelo indivíduo de expedientes diversos para conseguir que outras pessoas encarreguem-se de suas necessidades e responsabilidades pessoais. No Inventário ESEI, este fator será denominado Intitulação (INT), associado à crença do indivíduo de ter direitos ou privilégios especiais, que se revela na busca de ter as próprias necessidades supridas por outras pessoas, a serem manipuladas e colocadas a seu serviço.

Os itens agrupados no quinto fator não mostraram enquadramento teórico suficiente nas categorias descritas em modelos circumplexos. Estas cinco frases descrevem comportamentos de busca de superioridade social, principalmente através da proximidade com pessoas consideradas de status superior e do afastamento de atividades e relacionamentos no grupo de pares. Portanto, no Inventário ESEI, este fator será denominado Superioridade (SUP). A busca de superioridade é um conceito fundamental das ideias de Adler (1907/2005), referindo-se a busca compensatória de status superior associada a sentimentos de menos valia. Portanto, espera-se que escores elevados neste fator estejam relacionados à baixa autoestima e ansiedade social, sentimentos que o indivíduo tenta combater através de um estilo interpessoal caracterizado pelo controle da auto-apresentação e pela expressão de atitudes e comportamentos que ele considera associados a uma condição de superioridade social.

$\mathrm{O}$ sexto fator agrupou oito itens pertinentes às categorias hostil-dominante e hostil. As frases descrevem comportamentos agressivos, de retaliação por danos percebidos, intolerância a frustrações e de aversão por sentimentos de dependência ou subordinação. No Inventário ESEI, este fator será denominado Confrontação (CFR), onde escores elevados sugerem agressividade e competitividade bem como a busca por independência de opinião e comportamento.

$\mathrm{O}$ sétimo fator agrupou seis itens pertinentes às categorias submisso e amigável-submisso. Este fator será denominado Conformidade (CF), pois reúne descritores da necessidade de referências externas para guiar o comportamento, e de inclinações à disciplina e obediência. Acredita-se que escores elevados também estejam associados à preferência por situações sociais estruturadas por normas e com definição clara de papéis e responsabilidades.

$\mathrm{O}$ oitavo fator agrupou treze itens pertinentes às categorias amigável e socialmente interessado, que descrevem a preferência por atividades em grupo, associadas a sentimentos de confiança, inclusão e aceitação em relação aos demais. Este fator será denominado Pertencimento (PER), e espera-se que escores mais elevados indiquem estratégias interpessoais cooperativas e pacíficas.

A Tabela 2 apresenta as correlações entre as 8 escalas e duas facetas apresentadas pela escala de Reconhecimento. Destacam-se as correlações entre as seguintes escalas: (a) 
Magalhães, M. O. (2013). Escalas de Estilos Interpessoais (ESEI): Construção, Validade Fatorial e Consistência Interna.

Pertencimento e Cautela $(r=-0,58 ; p<0,01)$, que indica uma polarização entre comportamentos gregários e de esquiva/desconfiança; (b) Pertencimento e Superioridade ( $r=-0,41 ; p<0,01)$, que revela a contraposição entre comportamentos afiliativos e de distanciamento do grupo de pares; (c) Comando e Confrontação ( $r=0,46 ; p<0,01$ ), que revela a convergência entre comportamentos de liderança e de disputa pelo poder; (d) Comando e Intitulação $(r=0,38 ; p<0,01)$, que sugere que estratégias assertivas e manipulativas de controle do ambiente convergem em suas motivações de poder; (e) Reconhecimento e Conformidade ( $r=0,44 ; p<0,01)$, onde comportamentos auto-afirmativos e de conformidade/submissão convergem na busca pelo atendimento de expectativas externas de desempenho. As facetas da escala Reconhecimento, nomeadas Busca de aprovação e Busca de Sucesso revelaram direções contrárias em seu relacionamento com as escalas Cautela e Pertencimento. A faceta Busca de Aprovação obteve correlação negativa com Pertencimento $(r=-0,15 ; p<0,01)$ e positiva com Cautela $(r=0,16 ; p<0,01)$ sugerindo que esteja associada a maior insegurança interpessoal e menor disposição para relações cooperativas em comparação a faceta Busca de Sucesso, pois esta última apresentou correlação positiva com Pertencimento $(r=0,13 ; p<0,01) \mathrm{e}$ negativa com Cautela $(r=0,20 ; p<0,01)$. A correlação encontrada entre Busca de Sucesso e Comando $(r=0,31$; $p<0,01)$ sugere que a motivação para ocupar posições de liderança ou influência interpessoal pode estar associada com a busca do reconhecimento por realizações pessoais. Por fim, a análise da matriz de correlações ente as oito escalas permite concluir que a mesma não corresponde aos critérios para um modelo circumplexo (Gurtman, 2009). Por outro lado, dada a convergência correlacional entre escalas, foram explorados fatores de segunda ordem.

Tabela 2

Correlações (Pearson) entre as Escalas do Inventário ESEI $(N=971)$

\begin{tabular}{|c|c|c|c|c|c|c|c|c|c|}
\hline $\begin{array}{l}\text { Escalas do } \\
\text { Inventário ESEI }\end{array}$ & CA & $\mathrm{CM}$ & $\mathrm{BR}$ & INT & SUP & CFR & $\mathrm{CFO}$ & PER & BAP \\
\hline Cautela & - & & & & & & & & \\
\hline Comando & $0,18^{* *}$ & - & & & & & & & \\
\hline Reconhecimento & $-0,05$ & $0,31^{* *}$ & - & & & & & & \\
\hline Intitulação & $0,19^{* *}$ & $0,38^{* *}$ & $0,16^{* *}$ & - & & & & & \\
\hline Superioridade & $0,29^{* *}$ & $0,20^{* *}$ & $0,24^{* *}$ & $0,10^{* *}$ & - & & & & \\
\hline Confrontação & $0,17^{* *}$ & $0,46^{* *}$ & $-0,04$ & $0,24^{* *}$ & $0,13^{* *}$ & - & & & \\
\hline Conformidade & $-0,22^{* *}$ & $-0,06$ & $0,44^{* *}$ & $-0,03$ & $0,10^{* *}$ & $-0,24^{* *}$ & - & & \\
\hline Pertencimento & $-0,58^{* *}$ & $0,07^{*}$ & $-0,01$ & $-0,09^{* *}$ & $-0,41^{* *}$ & $0,09^{* *}$ & $0,08^{* *}$ & - & \\
\hline B. Aprovação & $0,16^{* *}$ & $0,18^{*}$ & $0,80^{* *}$ & $0,23^{* *}$ & $0,26^{* *}$ & $-0,03$ & $0,31^{* *}$ & $-0,15^{* *}$ & - \\
\hline B. Sucesso & $-0,20^{* *}$ & $0,31^{* *}$ & $0,88^{* *}$ & $0,07^{*}$ & $0,16^{* *}$ & 0,01 & $0,39^{* *}$ & $0,13^{* *}$ & $0,53^{* *}$ \\
\hline
\end{tabular}

Nota: $* \mathrm{p}<0,05 ; * * \mathrm{p}<0,01$.

A análise fatorial de segunda ordem com rotação Direct Oblimin resultou em uma solução de três fatores que explicaram $65 \%$ da variância. O primeiro fator agrupou as escalas de interesse social (carga negativa), cautela e superioridade, com cargas fatoriais entre 0,64 e 0,90 . O segundo fator agrupou as escalas busca de reconhecimento e conformidade, com cargas fatoriais de 0,84 e 0,83 . O terceiro fator agrupou as escalas intitulação, comando e confrontação, com cargas fatoriais entre 0,62 e 0,85 .

A convergência fatorial das escalas pertencimento, cautela e superioridade indica uma dimensão de segunda ordem consistente teoricamente por descrever comportamentos relacionados a maior ou menor motivação do indivíduo para aproximar-se de outras pessoas com objetivos de afiliação, amizade e cooperação. No segundo fator convergiram medidas relacionadas à disposição para atender expectativas sociais, atingir ou superar padrões de desempenho, de obter reconhecimento e status por realizações. Por fim, no terceiro fator agruparam-se escalas associadas à obtenção de poder e influência interpessoal. Estes resultados revelaram uma correspondência não esperada com o modelo clássico da motivação humana de David McClelland (McClelland, 1985; McClelland \& Koestner, 1992). Na abordagem motivacional de McClelland, os fatores internos que orientam o comportamento são as motivações de realização/sucesso, afiliação e poder. A motivação de realização se revela na busca pelo domínio de tarefas complexas com alta valorização do feedback positivo por desempenho e contribuição ao grupo. A motivação de afiliação se mostra no estabelecimento de relacionamentos pessoais próximos, de companheirismo e apoio, onde as pessoas são mais importantes do que seu desempenho. E a motivação de poder refere-se ao desejo de influenciar, controlar ou exercer autoridade sobre outros, com inclinações para atividades competitivas e interesse 
por obter e manter posições de prestígio (McClelland \& Koestner, 1992).

De acordo com Winter, Jonh, Stewart, Klohnen e Duncan (1998) modelos motivacionais são úteis para o entendimento das classes de incentivos ou metas que orientam os comportamentos, enquanto a avaliação dos traços informa a maneira pela qual as metas são perseguidas em ações particulares. Nesta visão integrada, os motivos canalizam ou direcionam as manifestações estilísticas do comportamento. Observa-se que os motivos de afiliação e poder são os eixos fundamentais dos modelos circumplexos de estilos interpessoais. Digman (1997) concluiu que estas duas dimensões organizam o modelo dos cinco grandes fatores. Os resultados da presente pesquisa sugerem que as escalas do Inventário ESEI organizam-se em torno dos vetores motivacionais afiliação, poder e realização. Entende-se que as escalas de estilos interpessoais descrevem expressões comportamentais pertinentes ao atendimento destes três motivos fundamentais. Por exemplo, a elevada motivação de poder pode se expressar de diversas formas, desde comportamentos abertamente confrontadores até estratégias encobertas de manipulação representadas na escala intitulação.

\section{Considerações Finais}

Assim como a maioria das teorias psicológicas, a construção do Inventário ESEI partiu da premissa de que as experiências infantis têm um impacto profundo na vida adulta. Acredita-se que cada indivíduo processa estas primeiras experiências para criar uma maneira consistente de lidar com as demandas da vida (Adler, 1907/2005). O uso da memória autobiográfica como uma técnica projetiva de avaliação da personalidade tem uma longa história. No entanto, a operacionalização psicométrica destes conceitos ainda foi pouco explorada. O Inventário ESEI inaugura esta linha de investigação no contexto brasileiro.

Os resultados desta pesquisa indicam que o Inventário ESEI apresenta um conjunto de medidas que oferece uma cobertura satisfatória das categorias de estilo interpessoal descritas na literatura. No entanto, recomenda-se a continuidade dos estudos de validação de construto em suas diversas modalidades, a fim de corroborar e refinar o entendimento do significado psicológico das escalas encontradas, bem como explorar o seu potencial de aplicação em intervenções psicológicas.

\section{Referências}

Adler, A. (2005). The Collected Clinical Works of Alfred Adler: Volume 1. The Neurotic Character. Washington, DC: Alfred Adler Institute. (Original work published 1907)

Adler, A. (2010). What life could mean to you? New York: Martino Books (Original work published 1931)

Atance, C. M., \& O'Neill, D. K. (2005). The emergence of episodic future thinking in humans. Learning and Motivation, $36,126-144$.
Bakan, D. (1966). The duality of Human Existence: Isolation and Communication in Western Man. Boston, MA: Beacon Press.

Bipp, T., Steinmayr, R., \& Spinath, B. (2008). Personality and achievement motivation: Relationship among Big Five domain and facet scales, achievement goals, and intelligence. Personality and Individual Differences, 44(7), 1454-1464.

Boyatzis, R. E. (2008). Competencies in the 21 st century. Journal of Management Development, 27(1), 5-12.

Bruhn, A. R. (1995). Early memories in personality assessment. In J. N. Butcher (Ed.), Clinical Personality Assessment: Practical Approaches (pp. 278-301). New York: Oxford Press.

Buckner, R. L., \& Carroll, D. C. (2007). Self-projection and the brain. Trends in Cognitive Science, 11, 49-57.

Costello, A. B., \& Osborne, J. W. (2005). Best practices in exploratory factor analysis. Practical Assessment Research \& Evaluation, 10(7), 1-9.

Curlette, W., Kern, R., \& Wheeler, M. (1997). BASIS - A Inventory: Technical Manual. Highlands, UK: TRT.

De Muth, P.W., \& Bruhn, A. R. (1997). The use of the Early Memories Procedure in a psychotherapy group of substance abusers. International Journal of Offender Therapy and Comparative Criminology, 41, 24-35.

Digman, J. M. (1997). Higher-order factors of the Big Five. Journal of Personality and Social Psychology, 73, 1246-1256.

Gurtman, M. B. (2009). Exploring personality with the Interpersonal Circumplex. Social and Personality Psychology Compass, 3. doi: 10.1111/j.1751-9004.2009.00172.x

Hogan, R. (1982). A socioanalytic theory of personality. In M. M. Page (Ed.), Nebraska symposium on motivation (pp. 55-89). Lincoln, NE: University of Nebraska Press.

Horowitz, L. M. (2004). Interpersonal foundations of psychopathology. Washington, DC: American Psychological Association.

Kern, R. M., Gormley, L., \& Curlette, W. L. (2008). BASIS-A Inventory empirical studies: Research findings from 2000 to 2006. Journal of Individual Psychology, 64, 280-309.

Kiesler, D. J. (1996). Contemporary interpersonal theory and research: Personality, psychopathology and psychotherapy. New York: Wiley.

Kiesler, D. J., \& Schmidt, J. A. (2006). The Impact Message Inventory-Circumplex (IMI-C) Manual. Redwood City, CA: Mind Garden.

Leary, T. (2004). Interpersonal diagnosis of personality. Eugene, OR: Resource Publications (Original work published 1957)

McAdams, D. P. (1985). Power, intimacy and the life story: Personological inquiries into identity. Homewood, IL: Dow Jones-Irwin.

McClelland, D. C. (1985). Human Motivation. New York: Cambridge University Press.

McClelland, D. C., \& Koestner, R. (1992). The achievement motive. In C. P. Smith (Ed.), Motivation and personality: Handbook of thematic content analysis (pp. 143-152). Cambridge, MA: Cambridge University Press.

Neisser, U. (1982). Memory observed: Remembering in natural contexts. San Francisco, CA: Freeman.

Pang, J. S., Villacorte, M. A., Chin, Y. S., \& Morrison, F. J. (2009). Achievement motivation in the social context: Implicit and explicit hope of success and fear of failure predict memory for and liking of successful and unsuccessful peers. Journal of Research in Personality, 43(6), 1040-1052.

Pillemer, D. B. (2003). Directive functions of autobiographical memory: The guiding power of the specific episode. Memory, 11(2), 193-202. 
Pincus, A. L., \& Gurtman, M. B. (2006). Interpersonal theory and the interpersonal circumplex: Evolving perspectives on normal and abnormal personality. In S. Strack (Ed.), Differentiating Normal and Abnormal Personality (pp. 83-111). New York: Springer.

Rank, O. (1945). Will therapy and truth and reality. New York: Knopf.

Rego, A., \& Leite, E. (2003). Motivos de sucesso, afiliação e poder: Um estudo de validação do constructo no Brasil. Estudos de Psicologia (Natal), 8(1), 185-191.

Roberts, B. W., \& Wood, D. (2006). Personality development in the context of the neo-socioanalytic model of personality. In D. K. Mroczek \& T. D. Little (Eds.), Handbook of personality development (pp. 11-39). Mahwah, NJ: Erlbaum.

Schacter, D. L., \& Addis, D. R. (2007). The cognitive neuroscience of constructive memory: Remembering the past and imagining the future. Philosophical Transactions of the Royal Society - Biological Sciences, 362, 773-786.

Schacter, D. L., \& Slotnick, S. D. (2004). The cognitive neuroscience of memory distortion. Neuron, 44, 149-160.

Suddendorf, T., \& Busby, J. (2005). Making decisions with the future in mind: Developmental and comparative identification of mental time travel. Learning and Motivation, 36, 110-125.

Sullivan, H. S. (1953). The interpersonal theory of psychiatry. New York: Norton.

Tulving, E. (2002). Episodic memory: From mind to brain. Annual Review of Psychology, 53, 1-25.

Tulving, E. (2005). Episodic memory and autonoesis: Uniquely human? In H. S. Terrace \& J. Metcalfe (Eds.), The missing link in cognition: Origins of self-reflective consciousness (pp. 3-56). New York: Oxford University Press.

Wiggins, J. S. (1991). Agency and communion as conceptual coordinates for the understanding and measurement of interpersonal behavior. In W. M. Grove \& D. Ciccetti (Eds.), Thinking clearly about psychology: Vol. 2. Personality and psychopathology (pp. 89-113). Minneapolis, MN: University of Minnesota Press.

Wiggins, J. S. (1995). Interpersonal Adjective Scales Professional Manual. Odessa, FL: Psychological Assessment Resources.

Winter, D. G., John, O. P., Stewart, A. J., Klohnen, E. C., \& Duncan, L. E. (1998). Traits and motives: Toward an integration of two traditions in personality research. Psychological Review, 105, 230-250. 